\title{
Study on the Mechanism of Action of MicroRNA-140-5p in the Treatment of Autism by Regulating the Nuclear Factor Kappa B Signaling Pathway
}

\author{
XIAO XIAO, RONG AI ${ }^{1}$, Y. TIAN, NING MI², W. LIU, S. CHENG, NA QIAN AND X. ZHU*
}

Department of Pediatrics, The Affiliated Hospital of Guizhou Medical University, 'Department of Pharmacology, Guizhou Medical University, Guizhou 550004, '2Department of Cardiovascular Medicine, First People's Hospital of Qingzhen, Guiyang, Guizhou 551499, China

Xiao et al.: Study on the Mechanism of Action of MicroRNA-140-5p in the Treatment of Autism

\begin{abstract}
To study the effect and mechanism of microRNA-140-5p on inflammatory factors in autistic rats induced by valproate. 50 Sprague-dawley rats at $12.5 \mathrm{~d}$ of pregnancy were randomly selected and divided into groups. The valproate group (10 rats) was intraperitoneally injected with valproate $(600 \mathrm{mg} / \mathrm{kg})$; the valproate+microRNA-negetive control group was intraperitoneally injected with valproate $(600 \mathrm{mg} / \mathrm{kg})$ and microRNA-negetive control; valproate+microRNA-140-5p group, intraperitoneal injection of valproate (600 mg/kg) and microRNA-140-5p; valproate+microRNA-140-5p+phorbol 12-myristate 13-acetate group was intraperitoneally injected with valproate $(600 \mathrm{mg} / \mathrm{kg})$ and microRNA-140-5p and phorbol 12-myristate 13-acetate. The control group (10 rats) was intraperitoneally injected with the same amount of normal saline. The impact of injury, polymerase chain reaction and enzyme-linked immunosorbent assay methods to detect the impact of inflammation-related and nuclear factor kappa B-related genes and proteins expression. The results showed that valproate-induced autistic rats may cause death or malformation, microRNA-140-5p may alleviate the death or malformation caused by it and phorbol 12-myristate 13-acetate may reverse the therapeutic effect of microRNA-140-5p; valproate modeling will cause the weight loss of rats and the high expression of microRNA-140-5p can effectively alleviate the weight loss and the use of phorbol 12-myristate 13-acetate will cause weight loss; microRNA-140-5p can shorten the time of opening eyes for the first time in valproate mice and can improve the learning and memory ability of valproate rats and the pathological damage of brain tissue; microRNA-140-5p can also inhibit the activation of nuclear factor kappa B signal to achieve the effect of inhibiting the expression of inflammatory factors. MicroRNA-140-5p reduces the secretion of inflammatory factors in valproate-induced autistic rats by regulating the nuclear factor kappa $B$ signaling pathway.
\end{abstract}

Key words: MicroRNA-140-5p, valproate, autism, nuclear factor kappa B

Autism is a serious neurodevelopmental disorder. The symptoms of autism are diverse and neurodevelopmental disorders include social communication difficulties, language communication difficulties, repetition, increased stereotyped behaviors and limited interests ${ }^{[1,2]}$. Children with autism often use primitive and atypical gestures to communicate with others, but they have special communicative functions for children with autism. The reason why children with autism use this method is that these behaviors can arouse people's attention and can greatly meet their communication needs. The main complaint in the first clinic of children with clinical autism is speech impairment, so speech recognition impairment has received widespread attention. As autistic children have their particularities in the development of cognition, thinking and social abilities, their language barriers have distinctive characteristics. And the differences between individuals are very large. Therefore, it is of great significance to find the specific markers of the disease and to understand its treatment mechanism ${ }^{[3,4]}$.

*Address for correspondence

E-mail: yofanowo@163.com 
MicroRNA (miRNA) is a type of non-coding RNA molecule that exists widely in the biological world. Because it does not have an open reading frame, it cannot encode proteins. miRNA has a variety of biological functions and plays a vital role in cell growth, differentiation and embryonic development ${ }^{[5]}$. Research reports show that miRNAs are related to the occurrence of diseases, miRNAs play a regulatory function in diabetes, heart disease and human tumors ${ }^{[6,7]}$ and miRNAs can also be used as molecular markers for disease diagnosis and treatment. MiR-140-5p has physiological and pathological regulatory effects. It has regulatory functions on cell energy metabolism and malignant transformation of tumor cells.

In this study, Valproate (VPA) rats were used as experimental subjects to explore its expression changes in VPA-induced autism models and to clarify the role of miR-140-5p in the secretion of inflammatory factors in VPA-induced autism models, in order to clarify VPA. The molecular mechanism of inflammation in the induced autism model and molecular targeted treatment of autism provide reference.

\section{MATERIALS AND METHODS}

\section{Animal model preparation and grouping:}

Healthy adult Sprague-Dawley (SD) rats in the reproductive cycle, weight: male 280-290 g, 10, female 240-250 g, 20. Raised for several days under the conditions of periodic light (07:00-19:00), constant temperature of $24^{\circ}$ and constant humidity of $55 \%$. After adapting to the environment for $2 \mathrm{w}$, follow the method of Schneider ${ }^{[8]}$ and others. At 17:00, the male to female ratio was $2: 1$. The vaginal smear was checked at 8:00 the next day. The sperm was found on the $1^{\text {st }} \mathrm{d}$ of pregnancy (E1); the pregnant rat is kept in another cage. Divide the pregnant rats into 5 groups immediately, At E12.5 $\mathrm{d}$ in the VPA group, pregnant rats in the model group were intraperitoneally injected with sodium valproate $600 \mathrm{mg} / \mathrm{kg}$ (sodium valproate powder was mixed with physiological saline to make a $250 \mathrm{mg} /$ $\mathrm{ml}$ solution); pregnant rats in the VPA+miR-Negetive Control (NC) group were injected intraperitoneally with valproate sodium valproate $600 \mathrm{mg} / \mathrm{kg}$ (sodium valproate powder is mixed with physiological saline to make $250 \mathrm{mg} / \mathrm{ml}$ solution) and injected with miR$\mathrm{NC}$; pregnant rats in the VPA+miR-140-5p group were injected intraperitoneally with sodium valproate 600 $\mathrm{mg} / \mathrm{kg}$ (valproic acid sodium powder was made into $250 \mathrm{mg} / \mathrm{ml}$ solution with physiological saline) and miR140-5p was injected; pregnant rats in the VPA + miR140-5p+Phorbol 12-Myristate 13-Acetate (PMA) group were injected intraperitoneally with sodium valproate $600 \mathrm{mg} / \mathrm{kg}$ (sodium valproate powder for physiological Saline was prepared as a $250 \mathrm{mg} / \mathrm{ml}$ solution) and injected with miR-140-5p and PMA; pregnant rats in the control group were injected with the same amount of normal saline intraperitoneally. The offspring born by the mother mouse in the model group are recorded as the sodium valproate group (VPA group, $n=20$ ); the offspring born by the mother mouse in the VPA+miR$\mathrm{NC}$ group (VPA+miR-NC group, $\mathrm{n}=20$ ); $\mathrm{VPA}+\mathrm{miR}-$ 140-5p pups from female rat (VPA + miR-140-5p group, $\mathrm{n}=20$ ); $\mathrm{VPA}+\mathrm{miR}-140-5 \mathrm{p}+\mathrm{PMA}$ pups from female rat (VPA+miR-140-5p+PMA group, $\mathrm{n}=20$ ); the offspring born by mother rat in the control group were recorded as the saline group (control group, $n=20$ ); the day of birth was recorded as the $1^{\text {st }} \mathrm{d}$ after birth (P1).

\section{Comparison of the mortality and deformity rate of the five groups of offspring in 1 mo:}

After the five groups of rats have grown up for 1 mo, observe their status and record the mortality and deformity rate of the offspring.

\section{Comparison of the body weight of rats in each group:}

Weigh the weights of rats in each group at P7, P14, P21, P56 and P90.

\section{Eye-opening time measurement:}

The rats in each group were observed to open their eyes at P12, P13, P14, P15 and P16. Scoring criteria: 0 points-both eyes are open; 1 point- 1 eye is open; 2 points-both eyes are open.

\section{Morris water maze test to detect the effect of miR- 140-5p on the learning and memory ability of rats:}

The Morris water maze experiment is divided into the $1^{\text {st }} 5 \mathrm{~d}$ of positioning and navigation experiments and the $6^{\text {th }} \mathrm{d}$ of space exploration experiments. The water maze circular pool is divided into four quadrants and the escape platform is placed in one of the quadrants. A training experiment was carried out from $\mathrm{d} 1$ to $\mathrm{d} 5$ and the rats were trained once a day at a fixed time in the morning and afternoon. The rats were put into the water facing the pool wall and the time when the rat found the platform within $60 \mathrm{~s}$ and the swimming route before the platform were recorded. It was recorded as the escape latency and swimming distance of the rat. If the mouse cannot find the platform within $60 \mathrm{~s}$, the escape latency is recorded as $60 \mathrm{~s}$ and the experimenter guides the mouse to the platform and stays for $20 \mathrm{~s}$. Analyze the spatial learning ability of rat. After the 
positioning and navigation experiment is over, the platform is removed on the $6^{\text {th }} \mathrm{d}$ and the rat are placed in the pool from any entry point and the time the rat stay in the target quadrant and the number of times they cross the platform are recorded ${ }^{[9,10]}$.

\section{Haemotoxylin and Eosin (HE) staining to observe the effect of miR-1400-5p on pathological damage in rats:}

Rats are anesthetized and perfused into the heart; the brain tissue is removed, dehydrated, embedded and sliced, with a thickness of $7 \mu \mathrm{m}$. The sections were fixed with alcohol; stained with hematoxylin staining solution; differentiation with hydrochloric acid alcohol differentiation solution; ammonia returning to blue; staining with eosin staining solution; dehydrated with gradient alcohol and dried, mounted with neutral gum and covered with a cover glass. Observe the pathological damage of the brain tissue of each group of mice under a microscope at $10 \times 20$ times and take pictures to record ${ }^{[11,12]}$.

\section{Reverse Transcriptase Polymerase Chain Reaction (RT-PCR) analysis:}

In this section, we examined the expression of inflammation related genes and nuclear factor kappa B (NF- $\kappa \mathrm{B})$ related messenger ribonucleic acid (mRNA). The total RNA was isolated from rat brains using TRizol reagent (Invirtrogen), the tissue were converted complementary DNA (cDNA) by OneScript Reverse Transcriptase OneScript cDNA Synthesis Kit (Abm). $25 \mu \mathrm{l}$ Dream Taq PCR Master Mix (Abm), $1.5 \mu 1$ forward and reverse primer (Ribobio, Guangzhou), 2 $\mu \mathrm{l}$ cDNA and $20 \mu \mathrm{l}$ water nuclease free in amplification reaction mixture $(50 \mu \mathrm{l})$ and the PCR condition were as follows: $95^{\circ}\left(2 \mathrm{~min}\right.$, a cycle), $95^{\circ}(30 \mathrm{~s}), 58^{\circ}(30 \mathrm{~s}), 72^{\circ}$ $(1 \mathrm{~min}), 35$ cycles in total. Finally, $72^{\circ}(10 \mathrm{~min}$ a cycle). $\beta$-actin served as the control ${ }^{[13,14]}$.

\section{Enzyme-Linked Immunosorbent Assay (ELISA) method for determination of cytokine levels:}

The brain tissues of rats were collected and the levels of interleukin-6 (IL-6), IL-1 $\beta$, Tumour Necrosis Factor alpha (TNF $\alpha), N F-\kappa B(p 65), p-I \kappa B \alpha$ and NFKB inhibitor $\alpha(\mathrm{I} \kappa \mathrm{B} \alpha)$ in rat tissues were determined according to the method steps of the ELISA kit instructions ${ }^{[15,16]}$.

\section{Statistical analysis:}

All data are shown as mean standard \pm deviation. Graphpad 6.0 statistical software (GraphPad Software Inc., San Diego, CA, USA) was used for the statistical analyses. Student's t-test was performed to analyze difference between two groups. One-way analysis of variance was used to detect differences between more than two groups. And p value was $<0.05$ was considered significant.

\section{RESULTS AND DISCUSSION}

The control group died within 1 mo with 1 rat without deformity. The VPA group died within the same periods of time 7 and 4 were deformed. The lethality and teratogenicity of the miR-NC+VPA group were the same as that of the VPA group. There were 3 deaths in the miR-140-5p+VPA group and 2 malformations, which was significantly lower than the miR-NC+VPA group. 5 deaths and 3 malformations in the miR-140$5 p+V P A+P M A$ group (Table 1), the above results showed that VPA-induced autistic rats may cause death or malformation, miR-140-5p may alleviate the death or malformation caused by it and PMA may reverse the therapeutic effect of miR-140-5p.

At P7, there was no significant difference in body weight between the groups ( $>>0.05)$; after P14, the differences between the groups gradually showed that the VPA group was significantly lower in weight compared with the control group at P14 ( $<<0.05)$, Compared with miR$\mathrm{NC}+\mathrm{VPA}$, the weight of rats in the miR-140-5p+VPA group increased significantly $(\mathrm{p}<0.05)$, while the weight of rats in the miR-140-5p+VPA group in the miR140-5p+VPA+PMA group There was no significant difference; at P21, the weight of rats in the VPA group was significantly lower than that of the control group $(p<0.01)$. Compared with miR-NC+VPA, the weight of rats in the miR-140-5p+VPA group was significantly increased $(p<0.01)$, while the miR-140-5p+VPA+PMA group miR-140-5p+VPA group rats weight decreased $(p<0.05)$; at P56 and P90, the weight change trend of each group was the same as that of $21 \mathrm{~d}$, of which 90 d. The difference between the groups was the most significant, as shown in Table 2.

At P12, P13 and P14, the score of eye-opening time in the VPA group was lower than that of the control group ( $\mathrm{p}<0.05)$; the eye-opening time score of miR140-5p+VPA was higher than that of the miR-NC+VPA group; while the comparison between the miR-140$5 p+V P A+P M A$ group and the miR-140-5p+VPA group has significant differences at P12 and P13, and there is no significant difference between the two groups at $\mathrm{P} 14$; at P15, there is no significant difference between the other groups ( $\mathrm{p}>0.05)$. At P16 and later, the mice in each group opened their eyes completely. As shown in Table 3. 
TABLE 1: COMPARISON OF MORTALITY AND DEFORMITY RATES

\begin{tabular}{lcccc}
\hline Group $(\mathrm{n}=20)$ & Death $(\mathrm{n})$ & Deformity $(\mathrm{n})$ & Death rate $(\%)$ & Deformity rate $(\%)$ \\
\hline Control & 1 & 0 & 5 & 0 \\
VPA & 7 & 4 & 35 & 20 \\
miR-NC+VPA & 7 & 4 & 35 & 20 \\
miR-140-5p+VPA & 3 & 2 & 15 & 10 \\
miR-140-5p+VPA+PMA & 5 & 3 & 25 & 15 \\
\hline
\end{tabular}

TABLE 2: WEIGHT COMPARISON BETWEEN GROUPS

\begin{tabular}{lccccc}
\hline & Control & VPA & miR-NC+VPA & miR-140-5p+VPA & miR-140-5p+VPA+PMA \\
\hline P7 & $14.8 \pm 1.4$ & $13.48 \pm 1.6$ & $13.2 \pm 1.4$ & $13.9 \pm 1.2$ & $13.5 \pm 1.2$ \\
P14 & $28.5 \pm 1.9$ & $21.1 \pm 1.3^{*}$ & $20.7 \pm 1.6$ & $27.7 \pm 1.6^{\#}$ & $25.7 \pm 1.3$ \\
P21 & $39.8 \pm 4.7$ & $27.6 \pm 7.1^{* *}$ & $16.9 \pm 6.4$ & $38.9 \pm 4.2^{\text {\#\# }}$ & $27.7 \pm 4.1^{\text {\& }}$ \\
P56 & $178.1 \pm 7.4$ & $167.3 \pm 8.1^{* *}$ & $165.2 \pm 7.3$ & $177.8 \pm 6.9^{\# \#}$ & $160.6 \pm 5.9^{\text {\&ध }}$ \\
P90 & $302.7 \pm 15.2$ & $284.5 \pm 12.7^{* *}$ & $281.9 \pm 13.4$ & $300.9 \pm 14.7^{\# \#}$ & $284.5 \pm 13.2^{\text {\&氏 }}$ \\
\hline
\end{tabular}

Note: ${ }^{*} \mathrm{p}<0.05$ vs. control; ${ }^{* *} \mathrm{p}<0.01$ vs. con; ${ }^{*} \mathrm{p}<0.05$ vs. miR-NC+VPA; ${ }^{\#} \mathrm{p}<0.01$ vs. miR-NC+VPA; ${ }^{\star} \mathrm{p}<0.05$ vs. miR-140-5p+VPA

TABLE 3: COMPARISON OF EYE OPENING IN EACH GROUP

\begin{tabular}{lccccc}
\hline & Control & VPA & miR-NC+VPA & miR-140-5p+VPA & miR-140-5p +VPA+PMA \\
\hline P12 & $0.60 \pm 0.69$ & $0.00 \pm 0.00^{*}$ & $0.00 \pm 0.00$ & $0.40 \pm 0.43^{\#}$ & $0.00 \pm 0.00^{\text {ध }}$ \\
P13 & $1.40 \pm 0.85$ & $0.00 \pm 0.00^{* *}$ & $0.00 \pm 0.00$ & $1.20 \pm 0.92^{\# \#}$ & $0.50 \pm 0.44^{\text {\&氏 }}$ \\
P14 & $1.70 \pm 0.56$ & $1.00 \pm 0.58^{*}$ & $1.00 \pm 0.47$ & $1.50 \pm 0.46^{\#}$ & $1.20 \pm 0.37$ \\
P15 & $2.00 \pm 0.00$ & $1.70 \pm 0.45$ & $1.80 \pm 0.56$ & $2.00 \pm 0.00$ & $1.90 \pm 0.32$ \\
P16 & $2.00 \pm 0.00$ & $2.00 \pm 0.00$ & $2.00 \pm 0.00$ & $2.00 \pm 0.00$ & $2.00 \pm 0.00$
\end{tabular}

Note: ${ }^{*} \mathrm{p}<0.05$ vs. control; ${ }^{* *} \mathrm{p}<0.01$ vs. con; ${ }^{*} \mathrm{p}<0.05$ vs. miR-NC+VPA; ${ }^{\# \#} \mathrm{p}<0.01$ vs. miR-NC+VPA; ${ }^{\natural} \mathrm{p}<0.05$ vs. miR-140-5p+VPA

In the Morris water maze experiment, compared with the blank control group, the escape latency and swimming distance of the VPA group were significantly increased and the number of crossing platforms and the percentage of stay time in the target quadrant were significantly reduced $(\mathrm{p}<0.05$ or $\mathrm{p}<0.01)$. Compared with the miR-NC+VPA group, the miR-140-5p+VPA group had significantly shorter escape latency and swimming distance and significantly increased the number of platform crossings and the percentage of stay time in the target quadrant; compared with the miR-140-5p+VPA group, miR-140-5p+VPA+PMA's escape latency and swimming distance are relatively prolonged and the number of crossing platforms and the percentage of stay time in the target quadrant are reduced; the above results indicate that the miR-140$5 p+V P A$ group can improve the learning and memory ability of VPA rats, as shown in fig. 1.

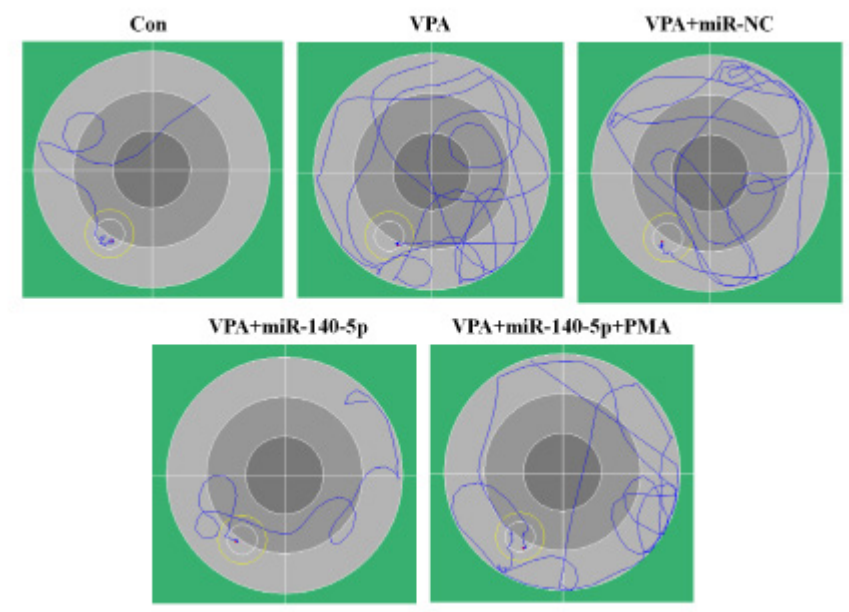

Fig. 1: Effects of miR-140-5p on the learning and memory function of VPA rats 
The HE staining results shows (fig. 2) that in the blank control group, the cells in the Carbonic Anhydrase 3 (CA3) area of the hippocampus of rats are evenly colored and the brain tissue cells are clearly and densely distributed, uniformly arranged, full cell bodies, clear nuclear membranes, complete cell structures and clear outlines. Compared with the blank control group, the cells in the hippocampal CA3 area of the VPA group are disorderly arranged with large gaps, irregular, lighter coloration, cell membranes and nuclear membranes are not clear. Compared with miR-NC+VPA, the brain tissue cells in the miR-140-5p+VPA group are clearly and densely distributed, evenly arranged and the cell body is full, that is, miR-140-5p can improve the brain tissue damage of VPA rats, while miR-140$5 p+V P A+P M A$ and miR-140-5p. Compared with miR140-5p+VPA, the arrangement is relatively disordered and the gap is large, irregular and the coloring is lighter. The above results indicate that PMA may reverse the therapeutic effect of miR-140-5p on the brain tissue of VPA rats.

In order to verify whether miR-140-5p can inhibit the expression of inflammatory factors and explore its relationship with the NF- $\mathrm{KB}$ signaling pathway, we used RT-PCR to detect the expression of related genes (fig. 3) and ELISA method to detect the expression of related proteins (Table 4). The results showed that compared with the control group, the expression of miR-140-5p in the brain of the VPA group was significantly reduced $(\mathrm{p}<0.01)$, miR-140-5p could significantly increase its expression $(p<0.01)$ and PMA has no regulatory effect $(p>0.05)$; at the same time, we detected the expression of inflammation-related factors including IL-6, IL-1 $\beta$ and TNF- $\alpha$. The results showed that compared with the control group, the expression of IL- 6 , IL- $1 \beta$, TNF- $\alpha$ were significantly increased $(\mathrm{p}<0.01)$; Compared with miR-NC+VPA, IL- 6 , IL-1 $\beta$ and TNF- $\alpha$ in the brains of rats in the miR-140-5p+VPA group were significantly reduced $(p<0.01)$. The above results indicate that miR$140-5 p$ can inhibit the expression of IL-6, IL-1 $\beta$, TNF- $\alpha$ and other inflammatory factors. Compared with miR140-5p+VPA, the expression of inflammatory factors has increased in miR-140-5p+VPA+PMA group. Therefore, we speculate that miR-140-5p inhibits the expression of inflammatory factors may be related to the NF- $\kappa B$ signaling pathway. The results showed that compared with the control group, the p65 and p-IкB $\alpha$ protein levels in the VPA group increased; miR-140$5 \mathrm{p}$ can significantly inhibit the $\mathrm{p} 65$ and $\mathrm{p}-\mathrm{I} \kappa \mathrm{B} \alpha$ protein levels (fig. 3 and Table 4); The protein expression levels of $\mathrm{p} 65$ and $\mathrm{p}-\mathrm{I} \kappa \mathrm{B} \alpha$ increased in the miR-140$5 p+V P A+P M A$ group. NF- $\kappa B$ signal activator PMA increases the level of NF- $\kappa B$ signal activation and partially reverses the inhibitory effect of miR-140$5 p$ on NF- $\kappa B$ signal. The above results indicated that miR-140-5p can inhibit the activation of NF- $\kappa B$ signal to achieve the effect of inhibiting the expression of inflammatory factors.

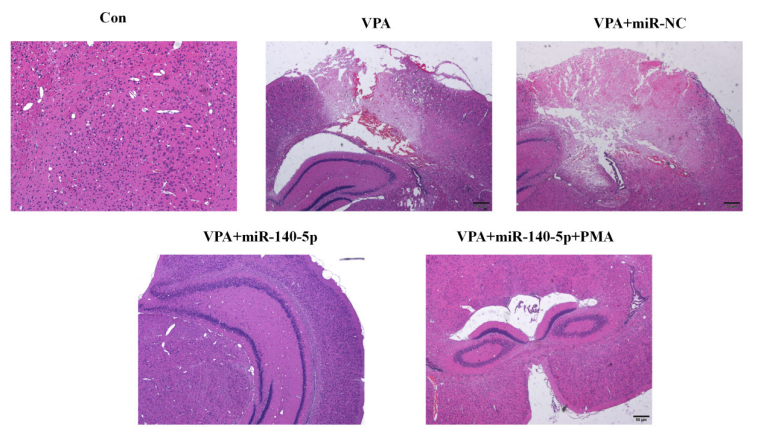

Fig. 2: HE staining to observe the effect of miR-140-5p on brain damage in autistic rats

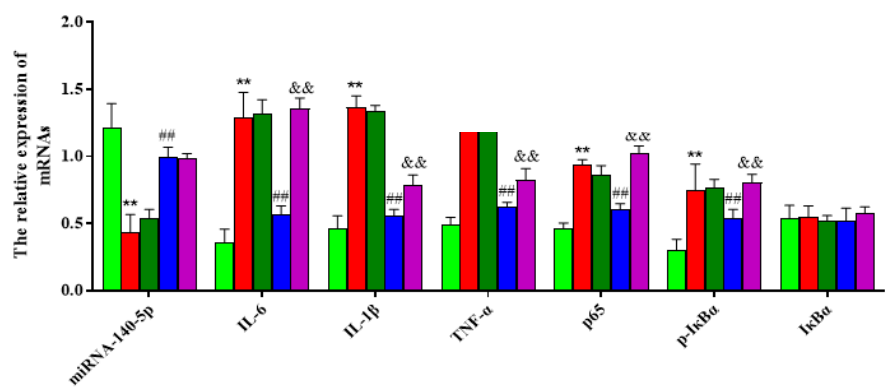

Fig. 3: The expression of IL-6, IL-1 $\beta$, TNF- $\alpha$, p65, p-IкB $\alpha$ and IкB $\alpha$ related genes were detected by RT-PCR Note: *p<0.05 vs. con; **p<0.01 vs. con; ${ }^{*} p<0.05$ vs. miR-NC+VPA; ${ }^{*} p<0.01$ vs. miR-NC+VPA; ${ }^{*} p<0.05$ vs. miR-140-5p+VPA,

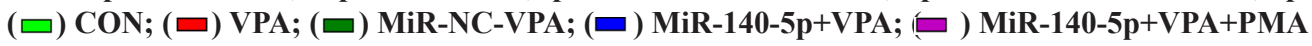


TABLE 4: VPA RATS TRANSFECTED WITH miR-140-5P, THE CONTENTS OF IL-6, IL-1B AND TNF- $\alpha$ IN THE BRAIN (pg/ml, xts)

\begin{tabular}{lccccc}
\hline Group & Control & VPA & miR-NC+VPA & miR-140-5p+VPA & $\begin{array}{c}\text { miR-140- } \\
5 p+V P A+P M A\end{array}$ \\
\hline IL-6 & $26.84 \pm 2.17$ & $35.76 \pm 2.03^{*}$ & $36.89 \pm 2.28$ & $29.54 \pm 2.30^{\#}$ & $38.61 \pm 2.08^{\natural}$ \\
IL-1B & $13.95 \pm 1.18$ & $22.81 \pm 1.24^{*}$ & $23.53 \pm 1.85$ & $17.38 \pm 1.42$ & $20.95 \pm 1.32$ \\
TNF-a & $5.15 \pm 0.40$ & $63.21 \pm 3.27^{* *}$ & $62.98 \pm 4.46$ & $12.45 \pm 1.23^{\# \#}$ & $28.45 \pm 2.19^{\natural}$ \\
p65 & $32.12 \pm 3.27$ & $51.23 \pm 5.34^{*}$ & $56.29 \pm 4.32$ & $40.27 \pm 3.28^{\#}$ & $52.29 \pm 5.62^{\natural *}$ \\
P-IKBa & $18.27 \pm 2.34$ & $45.39 \pm 4.69^{*}$ & $43.58 \pm 6.42$ & $33.59 \pm 3.57^{\#}$ & $50.27 \pm 6.47^{\natural}$ \\
IKBa & $46.28 \pm 5.37$ & $47.24 \pm 8.32$ & $45.38 \pm 8.58$ & $46.27 \pm 6.74$ & $47.29 \pm 6.42$ \\
\hline
\end{tabular}

Note: ${ }^{*} \mathrm{p}<0.05$ vs. con; ${ }^{* *} \mathrm{p}<0.01$ vs. con; ${ }^{\#} \mathrm{p}<0.05$ vs. miR-NC+VPA; ${ }^{\# \#} \mathrm{p}<0.01$ vs. miR-NC+VPA; ${ }^{\circledR} \mathrm{p}<0.05$ vs. miR-140-5p+VPA

Mothers who take VPA in early pregnancy to treat psychosis or anti-epilepsy have a very high risk of autism in their offspring ${ }^{[17]}$. Simple injection of pregnant mice, VPA can cause behavioral changes in offspring and maternal exposure to VPA will reduce autism candidates gene expression ${ }^{[18]}$. Electrophysiological studies have shown that VPA offspring exhibit abnormal microcircuit connections, which may be related to the long-term functional pathway damage in autistic patients shown by magnetic resonance imaging studies. Therefore, this article uses pregnant SD rats to establish a VPA autism model for follow-up research ${ }^{[19,20]}$. In order to clarify the molecular mechanism of inflammation in the induced autism model and molecular targeted treatment of autism provide reference.

miRNA has many biological functions and plays an important role in human embryo development, tissue function maintenance and tissue aging ${ }^{[21]}$. miRNA affects tissue function by regulating cell growth, differentiation, movement and apoptosis ${ }^{[22-25]}$. At present, the regulation of miRNAs has been found in the development of human malignant tumors, nerve damage, diabetes and other diseases ${ }^{[26-28]}$. The expression of miRNAs changes during the progression of the disease, and can promote or inhibit the occurrence of diseases by influencing the function of cells. miR$140-5 p$ is a miRNA molecule that is currently found to be related to the occurrence of human diseases ${ }^{[29]}$. miR$140-5 p$ is related to inflammation, tissue repair ${ }^{[30,31]}$ and is involved in the progression of a variety of malignant solid tumors, arthritis, intervertebral disc inflammation, etc. Therefore, this article is based on miR-140-5p is a key indicator to examine its relationship with the treatment of autism. This experiment showed that the expression level of miR-140-5p decreased in VPAtreated autistic rats and upregulation of miR-140-5p could inhibit the secretion of inflammatory factors in VPA-treated autistic rats. miR-140-5p may have inhibit inflammation.
This experiment further explored the mechanism of miR-140-5p and found that up-regulation of miR-140$5 p$ can reduce the expression levels of $p 65$ and $p-I \kappa B \alpha$ proteins in VPA-induced rats. Both $\mathrm{p} 65$ and $\mathrm{p}-\mathrm{I} \kappa \mathrm{B} \alpha$ are the key factors of NF- $\mathrm{KB}$ signal transduction. The higher the expression level of both, the higher the activation degree of NF- $\kappa \mathrm{B}$ signal. NF- $\kappa \mathrm{B}$ is highly conserved in evolution and it mostly exists as a dimer in human tissues. p65 is an indispensable subtype of $\mathrm{NF}-\kappa \mathrm{B}$ to function. NF- $\kappa \mathrm{B}$ is a transcriptional regulator ubiquitous in the human body, which participates in the processes of immunity and inflammation. Previous studies have shown that $\mathrm{NF}-\kappa \mathrm{B}$ is negatively regulated by miR-140-5p and is involved in the occurrence of inflammatory injury of the intervertebral disc. The mechanism of miR-140-5p is related to NF- $\kappa$ B. The results of this experiment show that NF- $\kappa \mathrm{B}$ activator can reverse the inhibitory effect of miR-140-5p on the secretion of inflammatory factors in VPA-induced autism rats, suggesting that miR-140-5p regulates VPAinduced inflammatory factors in autistic rats secretion is related to NF- $\kappa B$ signaling.

In conclusion, miR-140-5p reduces the secretion of inflammatory factors in VPA-induced autistic rats by regulating the $\mathrm{NF}-\kappa \mathrm{B}$ signaling pathway.

\section{Acknowledgement:}

This work was supported by The Affiliated Hospital of Guizhou Medical University.

\section{Declaration of interest:}

The authors report no conflicts of interest.

\section{REFERENCES}

1. Famitafreshi H, Karimian M. Overview of the recent advances in pathophysiology and treatment for autism. CNS Neurol Disord Drug Targets 2018;17(8):590-4.

2. Vorstman JA, Parr JR, Moreno-De-Luca D, Anney RJ, Nurnberger Jr JI, Hallmayer JF. Autism genetics: Opportunities and challenges for clinical translation. Nat Rev Genet 2017;18(6):362-76. 
3. Peters BC, Wood W. Autism and equine-assisted interventions: A systematic mapping review. J Autism Dev Disord 2017;47(10):3220-42.

4. Ding HT, Taur Y, Walkup JT. Gut microbiota and autism: key concepts and findings. J Autism Dev Disord 2017;47(2):480-9.

5. Lin Y, Liang X, Yao Y, Xiao H, Shi Y, Yang J. Osthole attenuates APP-induced Alzheimer's disease through upregulating miRNA-101a-3p. Life Sci 2019;225:117-31.

6. Bruneau BG. The developmental genetics of congenital heart disease. Nature 2008;451(7181):943-8.

7. Hoadley KA, Yau C, Hinoue T, Wolf DM, Lazar AJ, Drill $\mathrm{E}$, et al. Cell-of-origin patterns dominate the molecular classification of 10,000 tumors from 33 types of cancer. Cell 2018;173(2):291-304.

8. Schneider T, Przewlocki R. Behavioral alterations in rats prenatally exposed to valproic acid: Animal Model of Autism. Neuropsychopharmacology 2005;30(1):80-9.

9. Pellegrini C, Daniele S, Antonioli L, Benvenuti L, D'Antongiovanni V, Piccarducci R, et al. Prodromal intestinal events in Alzheimer's disease (AD): Colonic dysmotility and inflammation are associated with enteric AD-related protein deposition. Int J Mol Sci 2020;21(10):3523.

10. Gao S, Zhang X, Song Q, Liu J, Ji X, Wang P. POLD1 deficiency is involved in cognitive function impairment in $\mathrm{AD}$ patients and SAMP8 mice. Biomed Pharmacother 2019;114:108833.

11. Hussein EA, Hair-Bejo M, Omar AR, Arshad SS, Hani H, Balakrishnan $\mathrm{KN}$, et al. Velogenic newcastle disease virus tissue tropism and pathogenesis of infection in chickens by application of in situ PCR, immunoperoxase staining and HE staining. Microb Pathog 2019;129:213-23.

12. Xiao H, Li H, Song H, Kong L, Yan X, Li Y, et al. Shenzao jiannao oral liquid, an herbal formula, ameliorates cognitive impairments by rescuing neuronal death and triggering endogenous neurogenesis in AD-like mice induced by a combination of $\mathrm{A} \beta 42$ and scopolamine. J Ethnopharmacol 2020;259:112957.

13. Nie J, Zhou M, Lü C, Hu X, Wan B, Yang B, et al. Effects of triptolide on the synaptophysin expression of hippocampal neurons in the $\mathrm{AD}$ cellular model. Int Immunopharmacol 2012;13(2):175-80.

14. Wang Y, Chen F, Wang P, Mana L, Sheng N, Huang S. Study on myelin injury of AD mice treated with Shenzhiling oral liquid in the PI3K/Akt-mTOR pathway. Int J Immunopathol Pharmacol 2020;34:2058738420923907.

15. Pang B, Zhao C, Li L, Song X, Xu K, Wang J, et al. Development of a low-cost paper-based ELISA method for rapid Escherichia coli O157:H7 detection. Anal Biochem 2018;542:58-62.

16. Lassen LB, Gregersen E, Isager AK, Betzer C, Kofoed RH, Jensen PH. ELISA method to detect $\alpha$-synuclein oligomers in cell and animal models. PloS One 2018;13(4):e0196056.

17. Markram H, Rinaldi T, Markram K. The intense world syndrome-an alternative hypothesis for autism. Front Neurosci 2007;1:6.

18. Rinaldi T, Silberberg G, Markram H. Hyperconnectivity of local neocortical microcircuitry induced by prenatal exposure to valproic acid. Cereb Cortex 2008;18(4):763-70.
19. Nicolini C, Fahnestock M. The valproic acid-induced rodent model of autism. Exp Neurol 2018;299:217-27.

20. Ornoy A, Weinstein-Fudim L, Ergaz Z. Prevention or amelioration of autism-like symptoms in animal models: Will it bring us closer to treating human ASD? Int J Mol Sci 2019;20(5):1074.

21. Cao T, Zhen XC. Dysregulation of miRNA and its potential therapeutic application in schizophrenia. CNS Neurosci Ther 2018;24(7):586-97.

22. Yue J. miRNA and vascular cell movement. Adv Drug Deliv Rev 2011;63(8):616-22.

23. Sur S, Steele R, Shi X, Ray RB. miRNA-29b inhibits prostate tumor growth and induces apoptosis by increasing bim expression. Cells 2019;8(11):1455.

24. Zhang J, Xu Y, Liu H, Pan Z. MicroRNAs in ovarian follicular atresia and granulosa cell apoptosis. Reprod Biol Endocrinol 2019;17(1):1-1.

25. Shi C, Huang F, Gu X, Zhang M, Wen J, Wang X, et al. Adipogenic miRNA and meta-signature miRNAs involved in human adipocyte differentiation and obesity. Oncotarget 2016;7(26):40830.

26. Ganju A, Khan S, Hafeez BB, Behrman SW, Yallapu MM, Chauhan SC, et al. miRNA nanotherapeutics for cancer. Drug Discov Today 2017;22(2):424-32.

27. Morquette B, Juźwik CA, Drake SS, Charabati M, Zhang Y, Lécuyer MA, et al. MicroRNA-223 protects neurons from degeneration in experimental autoimmune encephalomyelitis. Brain 2019;142(10):2979-95.

28. Beuzelin D, Kaeffer B. Exosomes and miRNA-loaded biomimetic nanovehicles, a focus on their potentials preventing type-2 diabetes linked to metabolic syndrome. Front Immunol 2018;9:2711.

29. Teng L, Meng R. Long non-coding RNA MALAT1 promotes acute cerebral infarction through miRNAs-mediated hs-CRP regulation. J Mol Neurosci 2019;69(3):494-504.

30. Wang Y, Shen S, Li Z, Li W, Weng X. MIR-140-5p affects chondrocyte proliferation, apoptosis and inflammation by targeting HMGB1 in osteoarthritis. Inflamm Res 2020;69(1):63-73.

31. Wang H, Lou C, Ma N. miR-140-5p alleviates the aggressive progression of Wilms' tumor through directly targeting TGFBR1 gene. Cancer Manag Res 2019;11:1641.

This is an open access article distributed under the terms of the Creative Commons Attribution-NonCommercial-ShareAlike 3.0 License, which allows others to remix, tweak, and build upon the work non-commercially, as long as the author is credited and the new creations are licensed under the identical terms

This article was originally published in a special issue,
"Diagnostic and Therapeutic Advances in Biomedical Research
and Pharmaceutical Sciences"
Indian J Pharm Sci 2021:83(5) spl issue "133-139"

